\title{
Rehabilitation bei axialen Spondyloarthritiden
}

\section{Rehabilitation in Axial Spondyloarthritides}

W. Mau' ${ }^{1}$ I. Ehlebracht-König ${ }^{2}$

${ }^{1}$ Institut für Rehabilitationsmedizin, Martin-Luther-Universität Halle-Wittenberg

2 Rehazentrum Bad Eilsen, Schwerpunktklinik für Orthopädie und Rheumatologie

\section{Lernziele}

$\nabla$

Für die Rehabilitation von Menschen, die an axialen Spondyloarthritiden erkrankt sind, soll dieser Beitrag folgende Kenntnisse vermitteln:

- rehabilitationsrelevante Diagnostik

- Rehabilitationsindikation

- Merkmale des Rehabilitationsteams

- Interventionen in der Rehabilitation

- langfristiger Rehabilitationsprozess

\section{Einführung}

\section{$\nabla$}

Die Spondyloarthritiden (SpA) sind durch Manifestationen am Achsenskelett („Spondyl“-itis), den peripheren Gelenken („Arthritis“) und den Sehnenansätzen (Enthesen) sowie die Assoziation mit dem HLA-B27-Antigen charakterisiert [1]. Subtypen der SpA kommen mit folgenden Prävalenzraten in Deutschland vor [1]:

- axiale SpA/ankylosierende Spondylitis (AS; Morbus Bechterew) als Prototyp der SpA: $0,3-0,5 \%$,

- SpA bei Psoriasis: 0,1-0,3\%,

- reaktive SpA $<0,1 \%$, SpA bei chronisch entzündlichen Darmkrankheiten: $<0,1 \%$,

- undifferenzierte/nicht radiografische axiale SpA: 0,2-0,5\%.

Als Sonderform gilt das sehr seltene SAPHO-Syndrom (SAPHO: Synovitis, Akne, Pustulose, Hyperostose, Osteitis). Die AS als häufigster Typ manifestiert sich bereits im frühen erwerbsfähigen Alter von durchschnittlich 26 Jahren. Damit sind i.d.R. über eine lange Lebensphase hinweg rehabilitative Elemente im Krankheitsmanagement zu bedenken [2]. Der Schwerpunkt dieses Beitrags liegt auf Leistungen zur medizinischen Rehabilitation von Menschen mit vornehmlich axialen Formen der SpA, einschließlich AS.

\section{Rehabilitationsrelevante Diagnostik \\ Diagnoseüberprüfung}

Bereits vor der Zuweisung und Aufnahme zur rheumatologischen Rehabilitation soll die SpA diagnostiziert und die Einrichtung mit entsprechenden Unterlagen informiert werden. Allerdings sind dort oft weitere Untersuchungen notwendig, um die Diagnose zu sichern sowie Einschränkungen und Ressourcen auf Basis der ICF (Internationale Klassifikation der Funktionsfähigkeit, Behinderung und Gesundheit) zu klären. Daraus werden therapeutische und sozialmedizinische Entscheidungen abgeleitet.

Deutliche Einschränkungen der Wirbelsäulenbeweglichkeit ( $\bullet$ Abb. 1) [3] und der (konventionell) radiologische Nachweis einer Sakroiliitis nach früheren Klassifikationskriterien der AS sind vielfach erst nach mehrjährigem Krankheits-

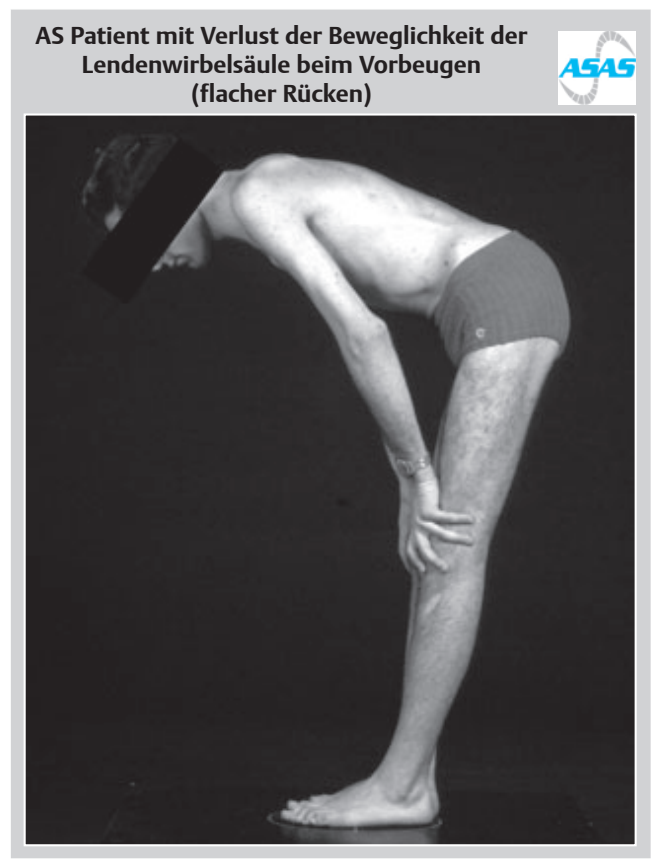

Abb. 1 Verlust der Lendenwirbelsäulenbeweglichkeit (nach [3]), mit freundl. Genehmigung von ASAS [www. asas-group.org]).

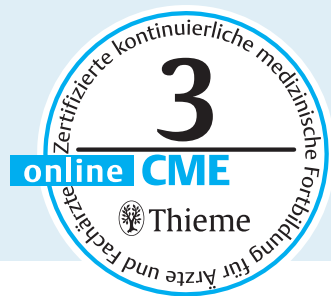

VNR

2760512013141210162

\section{Bibliografie}

DOI http://dx.doi.org/

10.1055/s-0032-1305165

Die Rehabilitation 2013;

52: 51-62

(c) Georg Thieme Verlag KG

Stuttgart · New York

ISSN 0034-3536

\section{Korrespondenzadresse}

Prof. Dr. med. Wilfried Mau

Institut für Rehabilitationsmedizin, Medizinische Fakultät,

Martin-Luther-Universität Halle-

Wittenberg

Magdeburger Straße 8

06097 Halle (Saale)

reha@medizin.uni-halle.de 
Abb.2 Magnetresonanztomografie der frühen Sakroiliitis (nach [3]), mit freundl. Genehmigung von ASAS [www.asasgroup.org]).

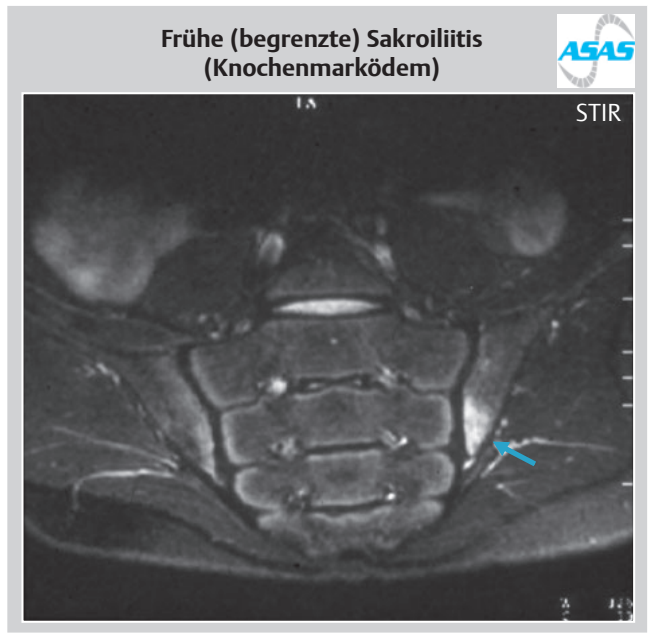

verlauf zu erwarten. Das ist spät für eine darauf gründende Zuweisung zur fachspezifischen Rehabilitation, die den Erhalt oder die Wiederherstellung einer guten Funktionsfähigkeit zum Ziel hat. Eine frühere diagnostische Zuordnung kann jetzt auf der Grundlage der Klassifikationskriterien 2009 der ASAS (Assessment of Spondyloarthritis International Society) für axiale SpA [1] vorgenommen werden: Hier genügt, neben klinischen Symptomen und Befunden, der Nachweis der aktiven Entzündung in der Magnetresonanztomografie ( $\bullet$ Abb.2) [3] oder des HLA-B27-Antigens (Infobox 1).

In der Rehabilitationseinrichtung ist die Diagnose anhand der SpA-Klassifikationskriterien 2009 der ASAS zu überprüfen. Diese beinhalten neben typischen klinischen SpA-Zeichen (Vor-)Befunde einer Sakroiliitis (Nachweis per Röntgen oder MRT) oder den Nachweis des HLA-B27-Antigens.

\section{ICF-Orientierung und Assessment}

Für die Planung und Durchführung der rehabilitativen Interventionen müssen alle Komponenten der ICF erfasst werden [4]. Die exemplarische Zuordnung wichtiger Symptome aus der Perspektive der von SpA Betroffenen und Assessments der Ärzte und Therapeuten zu diesen ICF-Komponenten zeigen $\bullet$ Abb. 3 und $\bullet$ Tab. 1 [5-9]. Informationen für die Dokumentation von Körperfunktionen und -strukturen ergeben sich aus der rheumatologischen Untersuchung. Für die rehabilitationsrelevante Erfassung der Aktivität und Teilhabe sowie der Kontextfaktoren sind komplexere generische und krankheitsspezifische Assessmentverfahren sinnvoll. Messwerte von Assessmentverfahren müssen stets kritisch im Gesamtkontext interpretiert werden, damit es nicht zu Fehlschlüssen kommt.

Die Verfahren können auch dazu dienen, die Alltags- und Teilhaberelevanz extravertebraler und extraartikulärer Manifestationen der SpA sowie somatischer und psychischer Komorbidität einzuschätzen und ggf. angemessen in den Rehabilita-

\section{Infobox 1}

Klassifikationskriterien 2009 der ASAS (Assessment of Spondyloarthritis International Society) für axiale Spondyloarthritiden (SpA) (nach [1]).

1. chronische Rückenschmerzen $\geq 3$ Monate, Patientenalter bei Beginn < 45 Jahre

2. Sakroiliitis (Nachweis über Röntgen oder MRT) plus $\geq 1$ weiteres SpA-Zeichen* oder Nachweis HLA-B27 plus $\geq 2$ weitere SpAZeichen*

*zusätzliche SpA-Zeichen:

$\checkmark$ entzündlicher Rückenschmerz (Morgensteifigkeit $>30 \mathrm{~min}$, Besserung bei Bewegung, nicht durch Ruhe, schmerzbedingtes nächtliches Erwachen, beidseits alternierender Gesäßschmerz)

- Arthritis

$>$ Enthesitis (Ferse)

$\rightarrow$ anteriore Uveitis

Daktylitis

- Psoriasis

- Kolitis bei chronisch entzündlicher Darmerkrankung

- gutes Ansprechen auf nicht steroidale Antirheumatika

Familiengeschichte für SpA

HLA-B27 positiv

erhöhtes C-reaktives Protein

tionsprozess einzubeziehen. Für die bei SpA erhöhte kardiovaskuläre Komorbidität können bspw. Interventionen wie kardiovaskuläres Training und edukative Elemente zur gezielten Reduktion von entsprechenden Risikofaktoren sinnvoll sein. Schließlich bilden die Ergebnisse der o.g. Diagnostik und des ICF-basierten Assessments durch das Rehabilitationsteam im Verlauf die Grundlage für die abschließende sozialmedizinische Begutachtung, bei der auch das qualitative und quantitative Leistungsbild bestimmt wird.

Einschränkungen durch die SpA, Komorbiditäten oder negative Kontextfaktoren und Ressourcen müssen zum einen im Rahmen der rheumatologischen Untersuchung, zum anderen über generische und krankheitsspezifische Assessment-Verfahren auf der Basis der ICF erhoben werden.

\section{Rehabilitationsindikation}

$\nabla$

Die individuelle Indikation für Leistungen zur medizinischen Rehabilitation bei SpA ergibt sich aus dem Rehabilitationsbedarf, der Rehabilitationsfähigkeit, den Rehabilitationszielen und der Rehabilitationsprognose. 


\begin{tabular}{|c|c|c|c|c|}
\hline \multirow{3}{*}{ 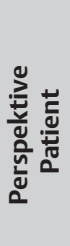 } & \multicolumn{4}{|c|}{ ICF Blatt } \\
\hline & $\begin{array}{l}\text { Schmerzen Rücken/Schulter } \\
\text { mit Bewegungseinschränkungen } \\
\text { Atemnot bei tiefer Einatmung } \\
\text { Erschöpfung/verminderte } \\
\text { psychische Belastbarkeit }\end{array}$ & $\begin{array}{l}\text { Einsc } \\
\text { Haus } \\
\text { Freiz } \\
\text { Mob } \\
\text { Steh }\end{array}$ & $\begin{array}{l}\text { jsaktivitäten: } \\
\text { nn, staubsaugen) } \\
\text { anges Sitzen) }\end{array}$ & $\begin{array}{l}\text { Einschränkungen der sozialen } \\
\text { Rolle: Beruf, Hausarbeit, } \\
\text { Freizeit }\end{array}$ \\
\hline & Struktur / Funktionen & & & Partizipation \\
\hline \multirow[t]{3}{*}{ 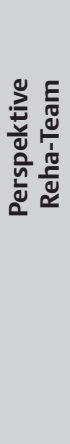 } & $\begin{array}{l}\text { Wirbelsäulenverformung } \\
\text { (Kyphosierung)/-ossifizierung } \\
\text { (auch Röntgen) } \\
\text { Bewegungseinschränkungen: } \\
\text { z.B. Finger-Boden-Abstand, } \\
\text { Hinterhaupt-Wand-Abstand, } \\
\text { Schobersches Maß, Neutral-Null- } \\
\text { Messung, verminderte Atembreite } \\
\text { Krankheitsaktivität } \\
\text { (Schmerzintensität, BASDA|lo), CRP) }\end{array}$ & $\begin{array}{l}\text { Funk } \\
\text { Gesu } \\
\text { (SF } 3\end{array}$ & insqualität & $\begin{array}{l}\text { Arbeitsunfähigkeitsdauer } \\
\text { Diskrepanz zwischen Fähigkeit } \\
\text { und beruflicher Anforderung für } \\
\text { einzelne Tätigkeiten wie Über- } \\
\text { Kopf-Arbeit, Stehen in gebückter } \\
\text { Haltung und Rumpfrotation } \\
\text { Screening beruflicher } \\
\text { Einschränkungen (SIMBO) d) }\end{array}$ \\
\hline & \multicolumn{3}{|c|}{ Personenbezogene Faktoren } & Umweltfaktoren \\
\hline & $\begin{array}{l}\text { Persönlichkeitsmerkmale, Bewc } \\
\text { Verhaltensmuster }\end{array}$ & & $\begin{array}{l}\text { Medikamente, } \\
\text { Soziale Faktoren }\end{array}$ & $\begin{array}{l}\text { edikamentöse Behandlung, } \\
\text { gen/Arbeitgeber, Partnerschaft }\end{array}$ \\
\hline
\end{tabular}

Abb. 3 ICF-Blatt mit Zuordnung der Hauptprobleme aus Sicht des Patienten und der zugrunde liegenden modifizierbaren und messbaren Mediatoren aus der Perspektive des Rehabilitationsteams zu den Komponenten des ICFModells [6] ( RPS-Form nach Steiner et al. [7], mit freundl. Genehmigung, a) Bath-Ankylosing-Spondylitis-Disease-Activity-Index [5], b) Funktionsfragebogen Hannover - Version Rücken [8], c) Bath-AnkylosingSpondylitis-Functional-Index [5], d) Screening-Instrument zur Feststellung des Bedarfs an medizinisch-beruflich orientierten Maßnahmen [9]).

Tab. 1 Ausgewählte Zielmediatoren der Rehabilitation nach $\bullet$ Abb. 3 mit Dokumentation konkreter Befunde und festgelegten Zielwerten bei der Aufnahmeuntersuchung sowie der erreichten Messwerte bei Entlassung, modifiziert nach [6, 7].

\begin{tabular}{|c|c|c|c|c|}
\hline ICF-Komponenten & Assessmentverfahren & Aufnahme & Entlassung: Ziel & Entlassung: erreicht \\
\hline $\begin{array}{l}\text { Körperstrukturen/-funktionen } \\
\text { Krankheitsaktivität }\end{array}$ & $\begin{array}{l}\text { Schmerzintensität gesamt } \\
\text { (NRS 0 - 10) } \\
\text { C-reaktives Protein } \\
\text { BASDAI }^{a}\end{array}$ & $\begin{array}{l}8 \\
15 \mathrm{mg} / \mathrm{l} \\
5,8\end{array}$ & $\begin{array}{l}4 \\
5 \mathrm{mg} / \mathrm{l} \\
2,6\end{array}$ & $\begin{array}{l}4 \\
10 \mathrm{mg} / \mathrm{l} \\
3,1\end{array}$ \\
\hline $\begin{array}{l}\text { Bewegungseinschränkungen } \\
\text { - Halswirbelsäule, Brustwirbel- } \\
\text { säule, Lendenwirbelsäule }\end{array}$ & $\begin{array}{l}\text { Neutral-Null-Messung } \\
\text { Hinterhaupt-Wand-Abstand } \\
\text { Finger-Boden-Abstand } \\
\text { Schober'sches Maß }\end{array}$ & $\begin{array}{l}\text { HWS: } \\
\text { Rotation re-li: } 35-0-35^{\circ} \\
8 \mathrm{~cm} \\
40 \mathrm{~cm} \\
10 / 12 \mathrm{~cm}\end{array}$ & $\begin{array}{l}\text { HWS: } \\
\text { Rotation re-li: } 50-0-50^{\circ} \\
6 \mathrm{~cm} \\
30 \mathrm{~cm} \\
10 / 13 \mathrm{~cm}\end{array}$ & $\begin{array}{l}\text { HWS: } \\
\text { Rotation re-li: } 45-0-45^{\circ} \\
6,5 \mathrm{~cm} \\
35 \mathrm{~cm} \\
10 / 12,5\end{array}$ \\
\hline - Thorax & Atembreite & $1,5 \mathrm{~cm}$ & $2,5 \mathrm{~cm}$ & $2,0 \mathrm{~cm}$ \\
\hline Aktivitäten & $\begin{array}{l}\text { FFBH-R } \\
\text { BASFI }^{\mathrm{b}}\end{array}$ & $\begin{array}{l}50 \% \\
5,5\end{array}$ & $\begin{array}{l}70 \% \\
7,0\end{array}$ & $\begin{array}{l}62,5 \% \\
6,4\end{array}$ \\
\hline Teilhabe & $\mathrm{SIMBO}^{\mathrm{d}}$ & 30 & - & - \\
\hline
\end{tabular}

a Bath-Ankylosing-Spondylitis-Disease-Activity-Index [5]; b Funktionsfragebogen Hannover - Version Rücken [8]; ' Bath-Ankylosing-Spondylitis-Functional-Index [5];

d Screening-Instrument zur Feststellung des Bedarfs an medizinisch-beruflich orientierten Maßnahmen [9]

\section{Rehabilitationsbedarf}

Rehabilitationsbedarf besteht u. a. dann, wenn alltagsrelevante und berufliche Aktivitäten oder die Teilhabe anhaltend beeinträchtigt sind und die ambulante Versorgung ausgeschöpft oder nicht ausreichend ist. Bei nicht radiografischer axialer SpA sowie AS mit Krankheitsdauern von unter 5 bzw. 5-10 Jahren bestehen ähnliche Einschränkungen der Alltagsaktivitäten mit Werten des BASFI (Bath Ankylosing Spondylitis Functional Index) von 2,5-3,1, Schmerzen von 4,8-5,1 und einer Krankheitsaktivität im BASDAI (Bath Ankylosing Spondylits Disease Activity Index) von 3,9 $-4,0$, jeweils auf einer Skala von $0-10[5,10]$. Ein ungünstiger Verlauf mit alltagsrelevanter Behinderung bei hoher Entzündungsaktivität und/ oder Strukturschäden an Wirbelsäule und peripheren Gelenken wird bei mehr als der Hälfte der AS-Patienten angenommen, v.a. wenn die in Infobox $\mathbf{2}$ zusammengefassten Risikofaktoren vorliegen. Frauen haben zwar später AS-typische ra- diologische Befunde, die weniger ausgedehnt sind und seltener zur Ankylosierung führen, sie leiden aber mehr unter Schmerzen und sind häufiger arbeitsunfähig als Männer [1].

\section{Infobox 2}

Risikofaktoren eines ungünstigen Verlaufs der ankylosierenden Spondylitis [1].

- Syndesmophyten bei der Erstvorstellung

- radiologische Veränderungen der Sakroiliakalgelenke in den ersten 2 Jahren

- erhebliche Sakroiliitis in der Magnetresonanztomografie (MRT)

- früher Beginn

- Hüftgelenksbeteiligung

- Beteiligung der Halswirbelsäule

- periphere Gelenkbeteiligung

- erhöhtes C-reaktives Protein (CRP) 
Einschränkungen der Teilhabe am Erwerbsleben werden durch die Arbeitsunfähigkeit und verminderte Erwerbstätigkeit deutlich: Im Alter von 18 - 65 Jahren waren in Deutschland 38\% der ambulant rheumatologisch betreuten AS-Patienten in den letzten 12 Monaten für durchschnittlich 65 Tage arbeitsunfähig; die indirekten Kosten beliefen sich auf 2336 Euro [11]. 22\% erhielten eine Erwerbsminderungsrente. Männliche AS-Patienten mit geringer Schulbildung (kein oder Hauptschulabschluss) und Wohnsitz in den neuen Bundesländern haben eine um ca. ein Drittel reduzierte Beschäftigungsrate gegenüber vergleichbaren Personen, die nicht an AS leiden [12].

\section{Rehabilitationsfähigkeit}

Die Fähigkeit, täglich an mehrstündigen Interventionen der Rehabilitation aktiv teilzunehmen, kann selten durch eine (noch) nicht ausreichend kontrollierte extrem hohe Entzündungsaktivität und sehr selten durch Frakturen der ankylosierten Wirbelsäule oder Organmanifestationen einer SpA, etwa eine Aorten- oder Lungenbeteiligung, eingeschränkt sein. Kontraindikationen gegen einzelne Maßnahmen wie intensive medizinische Trainingstherapie oder Ganzkörperbäder können sich aus der erhöhten kardiovaskulären Komorbidität, insbesondere der koronaren Herzkrankheit mit Herzinsuffizienz, ergeben. Die Motivation oder Motivierbarkeit der Rehabilitanden mit einer SpA ist in der Regel hoch.

\section{Rehabilitationsziele und -prognose}

Um realistische Rehabilitationsziele festzulegen, müssen die Beschwerden und die Assessmentergebnisse ( $\bullet$ Abb.3) in Verbindung gesetzt werden, und hinsichtlich ihrer Bedeutung für die alltäglichen und beruflichen Aktivitäten, die Teilhabe sowie ihre therapeutische Beeinflussbarkeit priorisiert werden. Konkrete messbare Ziele sind ggf. im Verlauf anzupassen ( $\bullet$ Tab. 1 und Infobox 3). Die Zielsetzung während der i.d.R. 3-wöchigen medizinischen Rehabilitation sollte in langfristige Ziel-, Zeit- und Interventionspläne eingebettet werden.

In einer randomisierten kontrollierten Studie führte eine 3-wöchige medizinische Rehabilitation zu besserer Funktionsfähigkeit und gleichzeitig höherer Kosteneffektivität [13, 14]. Auch in anderen Studien werden positive Effekte multimodaler Rehabilitationsprogramme nachgewiesen $[15,16]$. Diese positiven Daten erleichtern die individuelle Einschätzung der Rehabilitationsprognose.

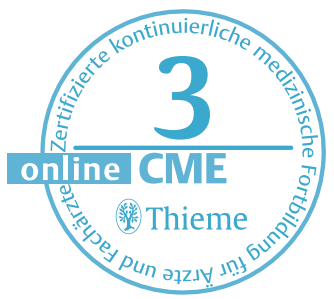

Die Rehabilitationsindikation wird v. a. durch SpAbedingte Schmerzen und Einschränkungen der Funktionsfähigkeit im Alltag und der Teilhabe (z. B. am Erwerbsleben) begründet. Rehabilitationsfähigkeit und -prognose bezüglich konkreter Ziele sind i.d.R. gut.

\section{Infobox 3}

Einordnung von exemplarischen Zielbereichen der Rehabilitation bei SpA in das ICF-Modell [6].

- Körperstrukturen/-funktionen:

> Verminderung der Krankheitsaktivität (Schmerzintensität, CRP; BASDAI)

- Verbesserung der Beweglichkeit aller Wirbelsäulenabschnitte

\ Kräftigung von Rücken- und Schultergürtelmuskulatur

- Erhalt bzw. Erhöhung der Atembreite psychische Entlastung

- Aktivität:

> Verbesserung der Alltagsaktivitäten (Haushaltstätigkeit, Unternehmungen in der Freizeit)

- Teilhabe:

- Anpassung des Arbeitsplatzes

Förderung privater sozialer Aktivitäten

- Kontextfaktoren:

> Stärkung der eigenen Ressourcen und Planung langfristiger fachkundiger Betreuung und Versorgung (Verbesserung der Schmerz- und Krankheitsbewältigung, Wissensvermittlung über Krankheitsbild und Interventionsmöglichkeiten mit dem Ziel der Therapieadhärenz und des Krankheitsmanagements)

\section{Rehabilitationsteam und -steuerung}

$\nabla$

Um die Rehabilitationsziele von SpA-Kranken zu erreichen, ist ein interdisziplinäres Team aus Ärzten, Physiotherapeuten, Sporttherapeuten, Ergotherapeuten, Pflegekräften, Ernährungsberatern, Psychologen, Sozialarbeitern und Rehabilitationsberatern erforderlich. Dieses Team wird von Ärzten koordiniert, die über rheumatologische, physikalisch-medizinisch-rehabilitative und sozialmedizinische Kompetenzen verfügen sollen. In Rehabilitationseinrichtungen mit Schwerpunkt auf entzündlich-rheumatischen Krankheiten werden zahlreiche Rehabilitanden mit SpA versorgt; dies fördert die Kompetenzbildung des Teams und erleichtert krankheitsspezifische Gruppeninterventionen, etwa standardisierte Patientenschulungen für SpA durch mehrere Professionen.

Die Rehabilitation von Menschen mit SpA erfolgt durch ein erfahrenes interdisziplinäres Team. Die koordinierenden Ärzte sollen über rheumatologische, physikalisch-medizinisch-rehabilitative und sozialmedizinische Kompetenzen verfügen. 


\section{Interventionen in der Rehabilitation \\ $\nabla$}

Die Interventionen sind bei der Behandlung der SpA sowohl krankheitsspezifisch als auch indikationsübergreifend ( Tab.2). Im Bereich der Bewegungstherapie, Ergotherapie und Physikalischen Therapie kommen viele indikationsspezifische Aspekte zum Zuge. Bei den psychologischen Interventionen und auch bei der medizinisch-beruflich orientierten Rehabilitation überwiegen indikationsübergreifende Therapieelemente [17]. Die Berücksichtigung von Kontextfaktoren spielt hier eine wichtige Rolle.

Die besten Wirksamkeitsnachweise für einzelne rehabilitative Interventionen bei $\mathrm{SpA}$ liegen für Medikamente, Bewegungstherapie und Schulungsprogramme vor [1]. Im Folgenden werden darüber hinaus auch die bedeutsamsten anderen Therapien dargestellt, für die positive Wirkungen analog zu anderen Indikationen angenommen oder die als innovative Ansätze der Rehabilitationspraxis verfolgt werden.

\section{Überprüfung/Anpassung der medikamen- tösen Therapie}

Bei einem nicht unerheblichen Teil der Patienten stellt sich bei der ausführlichen Anamnese heraus, dass sie die empfohlene Medikation (v.a. von NSAR) nicht so einnehmen, wie durch den ambulant behandelnden Arzt verordnet. Teilweise beruht dies auf Verständnisproblemen, zwischenzeitlich aufgetretenen Unverträglichkeiten, Ängsten oder anderen Vorbehalten auf Patientenseite. Es kann eine Überdosierung, eine Paralleleinnahme von 2 verschiedenen NSAR oder auch eine Unterversorgung bestehen. Neue Studienergebnisse deuten darauf hin, dass eine kontinuierliche Einnahme von NSAR gegenüber einer bedarfsweisen Einnahme zu einer geringeren radiologischen Progression führt [1]. Falls NSAR nicht ausreichend wirken (dies ist bei einem kleinen Teil der SpA-Patienten der Fall), ist der Einsatz von Biologika notwendig; ist eine Verbesserung der Funktions-, Arbeits- und Erwerbsfähigkeit durch deren Einnahme nachgewiesen [18, 19]. Aufgabe der behandelnden Klinik ist es, die Medikation und deren Verträglichkeit sowie die Laborparameter zu überprüfen. Dabei sind die Vorverordnungen des ambulant versorgenden Arztes zu berücksichtigen. Sind Anpassungen oder Veränderungen notwendig, müssen diese im Einzelfall mit den vorbehandelnden Ärzten abgestimmt werden. Dies muss auf jeden Fall erfolgen, wenn es um den Neueinsatz oder die Umstellungen von Biologika auf der Basis aktueller internationaler Empfehlungen [20] geht.

Die konsequente medikamentöse Therapie mit NSAR oder, bei deren unzureichender Wirkung, auch mit Biologika muss überprüft und ggf. -
Tab.2 Typische Interventionen bei axialer Spondyloarthritis nach ICF-Komponenten.

\begin{tabular}{|c|c|}
\hline $\begin{array}{l}\text { Problem- und Zielbereiche nach } \\
\text { ICF-Komponenten }\end{array}$ & Interventionen \\
\hline \multicolumn{2}{|l|}{ Körperstrukturen/-funktionen } \\
\hline Krankheitsaktivität & $\begin{array}{l}\text { medikamentös: NSAR; bei unzureichender } \\
\text { Wirkung Prüfung der Biologika-Indikation } \\
\text { physikalische Therapie: Thermotherapie } \\
\text { (Kälte, Wärme [z. B. zur muskulären Ent- } \\
\text { spannung]), Bindegewebsmassagen } \\
\text { psychologische Interventionen: Entspan- } \\
\text { nungsverfahren, Gespräche }\end{array}$ \\
\hline $\begin{array}{l}\text { Einschränkung der Wirbelsäulen- } \\
\text { beweglichkeit }\end{array}$ & $\begin{array}{l}\text { wirbelsäulengerechte Bewegungsanalyse/ } \\
\text { Bewegungstraining } \\
\text { Physiotherapie: Krankengymnastik (trocken, } \\
\text { im Wasser), klassische Massagen, medizini- } \\
\text { sche Trainingstherapie, Kletterwand, } \\
\text { Schwimmen, Schlingentisch, manuelle } \\
\text { Mobilisation } \\
\text { Ergotherapie }\end{array}$ \\
\hline Thoraxbeweglichkeit & Atemübungen, Thoraxmassagen \\
\hline $\begin{array}{l}\text { psychische Belastung, mangelnde Krank- } \\
\text { heitsbewältigung, nicht aktuelles Krank- } \\
\text { heitswissen (z. T. auch Kontextfaktoren) }\end{array}$ & $\begin{array}{l}\text { psychologische Einzelgespräche, Patienten- } \\
\text { schulungsprogramm, Entspannungsverfah- } \\
\text { ren }\end{array}$ \\
\hline \multicolumn{2}{|l|}{ Aktivität/Teilhabe } \\
\hline $\begin{array}{l}\text { Einschränkungen (der sozialen Rolle) } \\
\text { am Arbeitsplatz, im Haushalt etc. }\end{array}$ & $\begin{array}{l}\text { medizinisch-beruflich orientierte Rehabilita- } \\
\text { tion, Sozialdienst/Reha-Fachberatung zur } \\
\text { Erwerbstätigkeit } \\
\text { ergotherapeutische Unterstützung bei der } \\
\text { Planung der PKW-Umrüstung als Leistung zur } \\
\text { Teilhabe am Arbeitsleben sowie zum Ge- } \\
\text { brauch von Hilfsmitteln im Haushalt }\end{array}$ \\
\hline
\end{tabular}

in Rücksprache mit dem ambulant betreuenden

Arzt - angepasst werden.

\section{Bewegungstherapie}

Adäquate Bewegungsübungen und komplexe rehabilitative Interventionen werden häufig erst durch konsequente antiphlogistische und analgetische Medikation ermöglicht (i.d.R. mit NSAR oder Biologika). Ein intensives Bewegungsprogramm im rehabilitativen Setting führt gegenüber der alleinigen Biologikatherapie zur zusätzlichen Verbesserung der Beweglichkeit und Funktionsfähigkeit im Alltag [16, 21]. Gerade auch in der Rehabilitation sollen die Patienten durch positive Erfahrungen zu der Überzeugung geführt werden, dass die möglichst intensive Bewegung ein essenzieller Baustein in der lebenslangen Therapie dieser Erkrankung ist [20, 22, 23] ( $\bullet$ Abb.4). Sie dient der Verbesserung oder dem Erhalt der Beweglichkeit, der Muskelkraft, der Ausdauer und der Koordination.

Die Art der Bewegungstherapie richtet sich nicht nach der Diagnose, sondern nach den funktionellen Einschränkungen. Die Spannbreite reicht von (vorsichtiger) Einzelphysiotherapie bis zu einem intensiven Sportprogramm.

Beispiel 1: Ein Patient mit einer hohen Entzündungsaktivität, der gerade erst vor Beginn der Rehabilitation auf ein Biologikum eingestellt wurde,

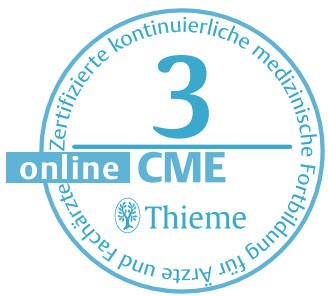




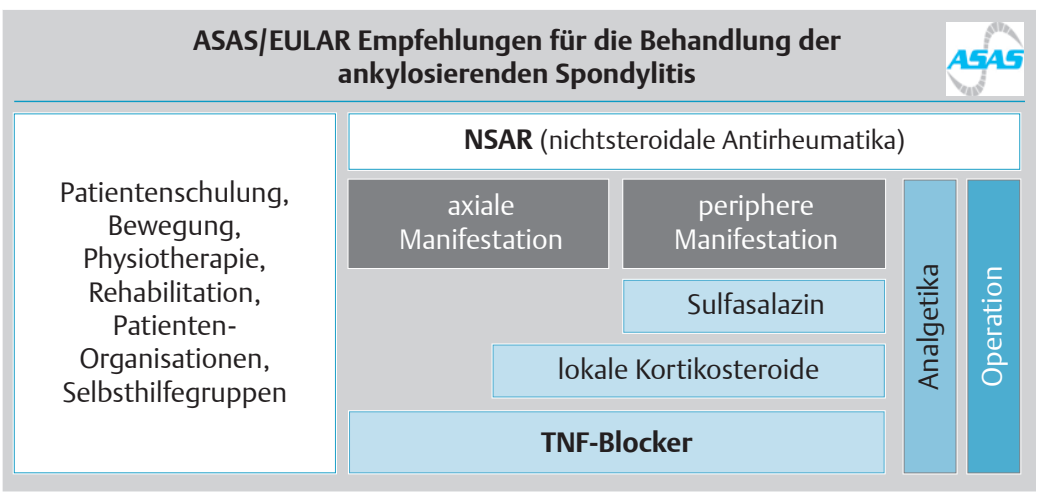

Abb.4 Empfehlungen der ASAS (Assessment of Spondyloarthritis International Society) und EULAR (European League Against Rheumatism) für die Behandlung der ankylosierenden Spondylitis (nach [3], NSAR: nicht steroidale Antirheumatika, mit freundl. Genehmigung von ASAS (www.asas-group.org) aus Zochling J et al. Ann Rheum Dis 2006; 65: 442 - 452, mit freundlicher Genehmigung der BMJ Publishing Group Ltd.).

mit einem BASDAI von 7, Schmerzbelastung von 8 (NRS 1-10) und erheblicher Schonhaltung sowie Bewegungseinschränkung im Halswirbelsäulenbereich. Ein solcher Patient wird einzelphysiotherapeutische Behandlungen erhalten, mit dem Ziel, die betroffenen Wirbelsäulenabschnitte zu mobilisieren und die Schmerzen zu linden. Er wird in der Lage sein, Übungen in der Wassergymnastik mitzumachen. Entscheidend sind jeweils gleitende Bewegungen ohne Stoß und Schlag. Bei weiterer Besserung kann das Programm vorsichtig ausgebaut werden.

Beispiel 2: Ein medikamentös gut eingestellter Patient mit einer gering ausgeprägten Krankheitsaktivität, BASDAI 3,5, Schmerzbelastung 3,8 und einer umschriebenen Bewegungseinschränkung im Lendenwirbelsäulenbereich (Schober'sches Maß 10/12 cm, bestehende syndesmophytäre Veränderungen). Ein solcher Patient kann neben einer auf das Krankheitsbild abgestimmten funktionellen Gruppengymnastik - durchaus ein umfangreiches, forderndes Sportprogramm mit Kraftausdauertraining an Geräten oder evtl. an der therapeutischen Kletterwand absolvieren. Als Ausdauersportarten kommen Walking, Nordic Walking, Joggen, Ergometertraining, Aqua-Jogging und Radfahren infrage.

Die Art der Bewegungstherapie richtet sich nach
der Krankheitsaktivität und den Funktionsein-
schränkungen.

Prinzipiell sind mobilisierende Übungen für die Wirbelsäule und den Thoraxbereich indiziert. Atemübungen sollten ebenfalls im Programm enthalten sein. Die Beweglichkeit der stammnahen Gelenke sollte unterstützt werden, da hierüber viele Einschränkungen der Wirbelsäulenfunktion kompensiert werden können. Auch die kardiopulmonale Fitness und die Muskelkraft sind in die therapeutischen Konzepte zu integrieren [22]. Selbstverständlich müssen Komorbiditäten im Herz-Kreislauf-Bereich, aber auch in Bezug auf die Wirbelsäule berücksichtigt werden.
Entscheidend für den Verlauf der Erkrankung und den Erhalt der Funktionskapazität ist die konsequente und langfristige körperliche Aktivität der Patienten. Hierbei müssen in der Rehabilitation motivierende Elemente in die konzeptionelle Gestaltung der Bewegungstherapie einbezogen werden [24]. Da es sich bei den von dieser Erkrankung Betroffenen gerade zu Beginn in der Regel um junge Erwachsene handelt, die sportliche Ambitionen haben können, sollten sie bezüglich günstiger Sportarten beraten werden. Als vorteilhaft sind Sportarten mit gleitenden Bewegungsabläufen zu werten (Schwimmen, Langlauf, Radfahren). Ein Verbot anderer Sportarten, die mit Stoß und Schlag einhergehen, z. B. Fußball oder Tennis, ist jedoch zu vermeiden. Wichtiger ist hier die Freude an der Bewegung - sie führt zu einer größeren Bindung an körperliche Aktivität. Letztlich erhöhen sich die Lebensqualität und der Trainingszustand im muskulären und kardiopulmonalen Bereich. Bei gutem Trainingszustand und Kenntnis der Bewegungsabläufe können Einschränkungen und Belastungen kompensiert werden.

Während der Rehabilitation haben Patienten die Möglichkeit, verschiedene Bewegungsarten kennenzulernen. In den zur Verfügung stehenden 3 Wochen machen die Patienten erste Erfahrungen, können erste Erfolge spüren oder erweitern das Spektrum bereits genutzter Möglichkeiten. Die positive Erfahrung und Motivation in der Rehabilitation ist für die anschließende Fortsetzung des aktiven Bewegungsverhaltens entscheidend. Hierfür stehen Nachsorgemöglichkeiten der Rehabilitationsträger zur Verfügung (s.u.), die zur Steigerung der Nachhaltigkeit genutzt werden sollten [25].

Für den Verlauf der Erkrankung und den Erhalt der Funktionskapazität ist die konsequente und langfristige körperliche Aktivität der Patienten entscheidend. Primäres Ziel ist daher eine dauerhafte Bindung an körperliche Aktivität.

\section{Ergotherapie}

In der ergotherapeutischen Versorgung werden Gelenk- und Wirbelsäulenschutz thematisiert. Ziel ist es, die Selbstständigkeit der Betroffenen im Alltag zu verbessern bzw. zu erhalten. Dazu werden Informationen und praktische Anleitungen für Alltag und Beruf individuell vermittelt. $\mathrm{Zu}$ Beginn wird von den Ergotherapeuten eine ADL-Diagnostik (ADL: Activity of Daily Living) durchgeführt, danach werden die jeweiligen Problembereiche herausgearbeitet. Bei Patienten, die keine wesentlichen Einsteifungen oder Funktionseinschränkungen haben, sind es Hilfen, die prophylaktisch eingesetzt werden. Bei deutlichen Einschränkungen werden kompensatorische Hilfsmittel notwendig. Dazu gehören Anziehhilfen für Schuhe und Strümpfe, Greifarme und vie- 
les mehr. Grundsätzlich knüpfen die Therapeuten an die bestehenden Ressourcen der Betroffenen an, häufig haben Betroffene Trickbewegungen entwickelt, die unterstützt oder korrigiert werden.

Bei Patienten mit SpA werden Alltagsbewegungen wie Stehen, Bücken, Heben, Sitzen, Liegen, Matratzenversorgung und Lagerung besprochen. Patienten mit einer SpA lagern sich aufgrund der Schmerzsituation nachts gern in eine Schonhaltung mit Hochlagerung des Oberkörpers und Unterpolsterung des Kopfes. Dadurch wird einer ungünstigen Fehlhaltung mit Versteifung in einer vermehrten Brustkyphose Vorschub geleistet. In der ergotherapeutischen Beratung werden - je nach Ausprägungsgrad der Erkrankung - Lagerungsalternativen gezeigt und erprobt, welche die Schonhaltung vermeiden.

Die Benutzung des Autos ist für die berufliche Integration und für die Mobilität in unserer Gesellschaft wichtig. Auch hier gibt es für Patienten mit SpA ergotherapeutische Unterstützungsmöglichkeiten, sei es das Beladen des Kofferraumes, die Sitzeinstellung oder auch die Spiegelversorgung. Insbesondere wenn beginnende oder fortgeschrittene Funktionseinschränkungen in der Halswirbelsäule vorliegen, benötigen die Patienten Hilfestellung bei der Ausgestaltung ihres Wagens.

Zum Lebensalltag gehören ebenfalls die bestehenden Arbeitsplätze. Wünschenswert ist es, wenn in der Klinik Modellarbeitsplätze bestehen (siehe auch Abschnitt „Medizinisch-berufliche Orientierung“). An einem PC-Arbeitsplatz können z.B. Schreibtischhöhe, Sitzhöhe, Einstellung des Monitors und die Bedienung der Maus individuell angepasst werden. Aber auch Werkzeuganpassung, Schutzkleidung, Sicherheitsschuhe usw. sind häufige Themen in der Beratungssituation.

In der Freizeit benutzen viele Betroffene das Fahrrad oder arbeiten im Garten. Auch hier gibt es Möglichkeiten der ergonomischen Anpassung: Die Patienten werden über verschiedene Lenkerformen unterrichtet, über die Möglichkeit der Spiegelausstattung bei eingesteifter Halswirbelsäule und über Sattelanpassungen. Dabei stehen Veränderungen im Vordergrund, die sie ohne wesentliche finanzielle Belastungen selber vornehmen können. Ebenso werden die Betroffenen bezüglich der Gartengeräte beraten.

Unter laufender Biologika-Therapie konnte bei AS-Patienten durch ein 6-stündiges ergotherapeutisches Programm mit individueller Information, ADL-Training mit Wirbelsäulen- und Gelenkschutz, Hilfsmitteleinsatz für Alltag und Arbeit und einem Hausübungsprogramm für die Wirbelsäule eine Verbesserung von Schmerzen, Funktionsfähigkeit (BASFI), Krankheitsaktivität (BASDAI), psychischem Befinden und Selbstmanagement erreicht werden [26].
In der Ergotherapie wird die Selbstständigkeit in Alltag und Beruf durch Information, individuelle Beratung und praktische Hilfen unterstützt. Häufige Themen sind Rückenlagerung, Spiegeleinstellung am Auto und Unterstützung mit anderen kompensatorischen Hilfsmitteln.

\section{Physikalische Therapie, Radon-Therapie}

Behandlungen aus der physikalischen Therapie können die Schmerzbelastung und Entzündungshemmung beeinflussen [23]; sie können sich auch auf bindegewebige und muskuläre Strukturen positiv auswirken. Die einzelnen Therapieverfahren müssen individuell angewendet werden, auch in Abhängigkeit von vorliegenden Komorbiditäten und von der subjektiven Verträglichkeit.

Zur Schmerzlinderung können Kälte- oder Wärmebehandlungen eingesetzt werden (lokale Kälte, Ganzkörperkältetherapie, Wannenbäder mit entsprechenden Zusätzen, Packungen mit unterschiedlichen Peloiden). Des Weiteren sind Ultraschallbehandlungen, Phonophoresen und Iontophoresen hilfreich, insbesondere bei Enthesitiden. Stangerbäder in Kombination mit Bewegungsübungen über 3 Wochen führen gegenüber Übungen ohne Stangerbäder kurzfristig zu deutlicheren Verbesserungen der Beweglichkeit, Funktionskapazität, Krankheitsaktivität und Lebensqualität [27]. Klassische Massagen werden eingesetzt, wenn es im Rahmen der Haltungsveränderung zu Überdehnungen oder Verkürzungen einiger Muskelabschnitte gekommen ist. Schmerzlindernd und entspannend können auch Sandliegen und Hydrojetliegen sein.

An einzelnen Standorten wird eine Radon-Therapie angeboten. Im Rahmen von 3-wöchigen stationären Rehabilitationsleistungen wurden bei ihrer Anwendung in einem Heilstollen gegenüber der Saunabehandlung jeweils deutlichere Besserungen der Alltagsfunktion (BASFI), Schmerzen und Morgensteifigkeit in der 4.-40. Woche nach Entlassung festgestellt [13].

Die Methoden der physikalischen Therapie können v. a. zur nichtmedikamentösen Schmerzlinderung genutzt werden. Insbesondere durch den positiven Einfluss auf bindegewebige und muskuläre Strukturen verbessern sie die Voraussetzungen für die Bewegungstherapie.

\section{Psychologische Betreuung}

Chronische Erkrankungen erfordern in Bezug auf die Krankheitsbewältigung von den Betroffenen eine erhebliche Anpassungsleistung und können mit entsprechenden psychischen Belastungen [28] einhergehen (Infobox 4). Etwa ein Drittel der SpA-Patienten hat Symptome einer Depression. Bei Mitgliedern einer britischen Selbsthilfegruppe wurden häufig Merkmale der Depression gefunden, ebenso wie die damit verbundenen ho-

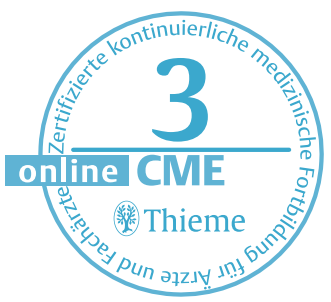


Tab.3 Beispiele für Aufgaben des psychologischen Dienstes während eines Rehabilitationsaufenthaltes.

\begin{tabular}{ll}
$\begin{array}{l}\text { Aufgabenfelder } \\
\text { Erkennen von psychischen Belastungen } \\
\text { und Störungen }\end{array}$ & Interventionsbeispiele \\
\hline $\begin{array}{l}\text { Unterstützung der Betroffenen im } \\
\text { Rahmen der Krankheitsbewältigung }\end{array}$ & $\begin{array}{l}\text { Psychoedukation } \\
\text { Entspannungsverfahren } \\
\text { Einzelgespräche }\end{array}$ \\
\hline $\begin{array}{l}\text { Unterstützung bei der Schmerz- } \\
\text { bewältigung }\end{array}$ & $\begin{array}{l}\text { Schmerzbewältigungskurse } \\
\text { Entspannungsverfahren }\end{array}$ \\
\hline $\begin{array}{l}\text { Steigerung der Therapiemotivation } \\
\text { Einzelgespräche }\end{array}$ \\
\hline $\begin{array}{l}\text { Hilfestellung bei Lebensstiländerungen, } \\
\text { Steigerung der Handlungskompetenz }\end{array}$ & $\begin{array}{l}\text { Psychoedukation } \\
\text { Einzelgespräche }\end{array}$ \\
\hline $\begin{array}{l}\text { Indikationsstellung für weitergehende } \\
\text { psychotherapeutische Behandlung }\end{array}$ & Stressbewältigungskurse \\
\hline $\begin{array}{l}\text { Dokumentation der Diagnostik und } \\
\text { Therapie }\end{array}$ & $\begin{array}{l}\text { Dokumentation gemäß dem Katalog } \\
\text { Therapeutischer Leistungen (KTL) }\end{array}$ \\
\hline
\end{tabular}

hen internen und geringen externen Kontrollüberzeugungen bezüglich der Bewältigung der körperlichen Erkrankung [29]. Bei der Stressbewältigung von AS-Patienten wurde vielfach das Wahrnehmungskonzept des „Herunterspielens“ festgestellt [30]. Das Ausmaß der psychischen Beeinträchtigung korreliert mit der Aktivität und Funktionseinschränkung bei SpA [31]. Vielfältige psychologische Angebote dienen in der medizinischen Rehabilitation dazu, die psychische Belastung zu reduzieren ( Tab.3). Interventionen, die auf Krankheitsbewältigung, Lebensstiländerung und Schmerzbewältigung abzielen, haben dabei einen hohen Stellenwert. Psychische Komorbiditäten führen zu einer verminderten Lebensqualität [32] und stellen zudem einen Risikofaktor für die Schmerzchronifizierung dar. Es ist wichtig, sie zu erkennen und zu behandeln.

Psychische Komorbidität ist ein wichtiger Risikofaktor für die Schmerzchronifizierung. In der psychologischen Betreuung steht die entsprechende Diagnostik im Vordergrund, sowie therapeutische Interventionen, die die Krankheits- und Schmerzbewältigung positiv beeinflussen und auf eine Lebensstiländerung abzielen.

\section{Patientenschulung}

Unter Patientenschulung versteht man den Einsatz von strukturierten und nach Möglichkeit standardisierten Schulungsprogrammen. Sie dienen der Informationsvermittlung zum Krankheitsbild und zu den Behandlungsmöglichkeiten. Sie fördern den Austausch von Betroffenen untereinander und fokussieren auf die Verbesserung der Selbstwirksamkeit und Handlungskompetenz. Schulungsprogramme werden diagnosebezogen oder problembezogen konzipiert. Sie sind ein wesentlicher Baustein im Rehabilitationsprozess [33]. Der Arbeitskreis Patientenschulung der Deutschen Gesellschaft für Rheumatologie

\section{Infobox 4}

Mögliche psychische Belastungen von Patienten mit chronischen körperlichen Erkrankungen [28].

- Irreversibilität und/oder Progredienz

- subjektive und/oder objektive Lebensbedrohung

- reduzierte körperliche Leistungsfähigkeit

- bedrohte körperliche Integrität und bedrohtes Selbstbild

- stigmatisierende Reaktionen des Umfeldes

- Bedrohung von sozialen Beziehungen

- chronische Schmerzen

- aversiv erlebte therapeutische Maßnahmen

- Abhängigkeit vom medizinischen System

(temporäre) Hospitalisierung

- Unvorhersagbarkeit des Krankheitsverlaufs

- begrenzte Lebensplanung und Zukunftsperspektive

(DGRh) bietet ein auf die SpA abgestimmtes Schulungsprogramm an, das umfangreich evaluiert wurde [34-36]. Es wird durch ein interdisziplinäres Team, bestehend aus Arzt, Psychologe, Ergotherapeut und Physiotherapeut, mit einem Zeitumfang von 6-mal 90 Minuten durchgeführt. Die Trainer werden durch ein spezielles Train-theTrainer-Seminar geschult und können interaktiv und durch Einsatz von verschiedenen Methoden das Curriculum in einer Kleingruppe von max. 10 -12 Teilnehmern umsetzen. Die Inhalte und die Methoden sind im Curriculum hinterlegt. Das Schulungsprogramm ist innerhalb der medizinischen Rehabilitation gut umsetzbar. Die Akzeptanz auf Patientenseite ist sehr hoch, die Auswirkungen auf die Krankheitsbewältigung und die gesundheitsökonomischen Aspekte sind belegt.

Patientenschulung beinhaltet kognitive, emotionale und handlungsrelevante Elemente. Sie führt zu einer Steigerung der Selbstwirksamkeit und zeigt langfristige positive Effekte im Selbstmanagement sowie bei den Krankheitskosten.

\section{Medizinisch-beruflich orientierte}

\section{Rehabilitation und Sozialberatung}

Verschiedene körperlich belastende Tätigkeiten sind als Risikofaktoren dafür anzusehen, dass die Erwerbstätigkeit bei SpA beendet wird [37]. Entsprechend wichtig ist es, sie im Rehabilitationsprozess angemessen zu beachten. Der Erwerbsbezug hat in den letzten Jahren in der medizinischen Rehabilitation immer weiter an Bedeutung gewonnen [17] und durch berufsbezogene Interventionen $\mathrm{zu}$ verbesserten Ergebnissen geführt [38].

Patienten mit SpA haben, bezogen auf den Beruf, sehr heterogene Bedürfnisse [9]. Bei einem jungen SpA-Patienten, der einen körperlich belasten- 
den Beruf ausübt und bei dem absehbar ist, dass er nicht bis zum Rentenalter in diesem Beruf arbeiten kann, kommt beispielsweise eine berufliche Neuorientierung infrage: Testungen und Beratungen können durchgeführt werden, und es werden mit dem Patienten Alternativen erarbeitet. Entscheidend ist, dass bei Entlassung eine berufliche Perspektive besteht und der Rehabilitand weiß, an wen er sich zur weiteren Umsetzung wenden kann. Bei längerem Krankheitsverlauf und bestehenden funktionellen Einschränkungen kann ein arbeitsplatzbezogenes Training einschließlich ergonomischer Beratung sinnvoll sein. Dieses Training wird von Physio- oder Ergotherapeuten durchgeführt, die eine entsprechende $\mathrm{Zu}-$ satzausbildung haben (Evaluation der funktionellen Leistungsfähigkeit nach Isernhagen). Es bezieht sich immer auf den aktuellen Arbeitsplatz und greift 3-5 entscheidende Bewegungsabläufe auf. Das Training kann mit einer internen Belastungserprobung gekoppelt sein.

Berufsbezogene Therapieelemente richten sich nach den empfundenen Arbeitsbelastungen, den funktionellen Einschränkungen und v.a. den beruflichen Kontextfaktoren der Rehabilitanden. Die Unterstützungsmöglichkeiten müssen während der Rehabilitation individuell abgestimmt und eine Weiterbetreuung durch die zuständigen Leistungsträger initiiert werden.

\section{Planung des langfristigen Reha-Prozesses}

Eine Rehabilitation von 3, gelegentlich 4 Wochen, stellt für die SpA-Patienten nur einen kleinen Zeitausschnitt im oft lebenslangen Krankheitsverlauf dar. Die medizinische Rehabilitation soll die Betroffenen zu einem aktiven Lebensstil motivieren. Sie müssen Informationen erhalten und Fertigkeiten erlernen, die sie in ihrem Lebensalltag dauerhaft integrieren können. Das können Selbstmanagementelemente sein, Veränderungen von Überzeugungen und Einstellungen, berufliche Anpassungen und vieles mehr.

Eine wesentliche Aufgabe der Rehabilitation ist es, bei den Betroffenen eine Bindung an regelmäßige körperliche Aktivität zu erzielen. Aus diesem Grund haben Rehabilitationskliniken die Möglichkeit, ein Nachsorgeprogramm für die Patienten zu verordnen. Von den Leistungsträgern werden spezielle bewegungsbezogene Nachsorgeprogramme angeboten. Im Funktionstraining und Reha-Sport gibt es in einigen Regionen eigenständige „Bechterew-Gruppen“, die i.d.R über die Deutsche Vereinigung Morbus Bechterew e.V. organisiert werden. Über diese sehr positiv zu bewertenden Angebote erhalten Betroffene gleichzeitig eine Anbindung an die Selbsthilfe. Bereits während der Rehabilitation sollen entsprechende Angebote mit dem Patienten besprochen und he-

rausgesucht werden, damit die körperliche Aktivität möglichst rasch nach der Rehabilitation fortgesetzt wird. Es sollen Ansprechpartner in der Selbsthilfe genannt werden, die neben Bewegungstraining vielfältige weitere Angebote vorhalten. Über die vielfältigen Möglichkeiten zur Unterstützung der (Re-)Integration in Beruf und Gesellschaft beraten die Gemeinsamen Servicestellen der Leistungsträger.

Benötigt der Betroffene weitergehende Unterstützung für die Integration im Arbeitsmarkt, so können Empfehlungen für ein breites Spektrum von „Leistungen zur Teilhabe am Arbeitsleben“ (LTA) im Arztbrief ausgesprochen werden. Außerdem können Ansprechpartner vermittelt werden, z.B. in den Auskunfts- und Beratungsstellen der Rentenversicherungen.

Am Ende der Rehabilitation gibt es Möglichkeiten, die Nachhaltigkeit auf motorisch funktioneller Ebene zu fördern, etwa durch Verordnung von Funktionstraining oder Rehabilitationssport. Die Integration im Erwerbsleben wird durch den Beratungsdienst der Rentenversicherung unterstützt.

\section{Interessenkonflikt}

$\nabla$

W. Mau hat eine Stiftungsprofessur für Rehabilitationsmedizin der Deutschen Rentenversicherung Mitteldeutschland und der Deutschen Rentenversicherung Bund. I. Ehlebracht-König ist Ärztliche Direktorin des Rehazentrums Bad Eilsen, Schwerpunktklinik für Orthopädie und Rheumatologie, der Deutschen Rentenversicherung Braunschweig-Hannover. Beide Autoren sind Vorstandsmitglieder der Deutschen Gesellschaft für Rehabilitationswissenschaften. Sie erklären, dass sie keinen Interessenkonflikt haben.

\section{Kernaussagen}

- Die medizinische Rehabilitation bei SpA beinhaltet vielfältige Aufgaben der Diagnostik und Therapie nach internationalen Klassifikationskriterien und Empfehlungen. Sie erfüllt wichtige Funktionen der Weichenstellung für die (Re-)Integration von SpA-Patienten in Beruf und Gesellschaft.

- Von zentraler Bedeutung ist das ärztlich koordinierte interdisziplinäre Team, das über Erfahrungen mit der ICF-orientierten Rehabilitation von SpA-Betroffenen verfügt.

- Die Kombination aus Bewegungstherapie, unterstützt durch eine leitliniengerechte medikamentöse Therapie, physikalischer Medi- zin und Ergotherapie sowie psychologischen Verfahren und interdisziplinären Patientenschulungen ermöglicht Verhaltensänderungen. Bedarfsgerecht werden die Therapieelemente berufsbezogen ausgerichtet.

- Durch positive Erfahrungen in der Rehabilitation sollen die Betroffenen erlernte Bewegungsaktivitäten lebenslang in ihren Alltag integrieren.

- Zur Förderung der Nachhaltigkeit können bei Bedarf Nachsorgeleistungen wie Funktionstraining oder Rehabilitationssport sowie Leistungen zur Teilhabe am Arbeitsleben eingeleitet werden. 


\section{Literatur}

1 Braun J, Rudwaleit M, Sieper J. Spondyloarthritiden. Internist 2011; 52: 657-670

2 Zochling J, van der Heijde D, Burgos-Vargas $R$ et al. ASAS/EULAR recommendations for the management of ankylosing spondylitis. Ann Rheum Dis 2006; 65: $442-452$

3 ASAS slide library. ASAS Assessment of SpondyloArthritis International Society. Im Internet: http:// www.asas-group.org/education.php?id=01 Stand: 02.05.2012

4 Ehlebracht-König I, Mau W. Rheumatologische Rehabilitation heute. Veranschaulichung an 2 Fallbeispielen. Z Rheumatol 2008; 67: 554-564

5 Haibel H, Rudwaleit M, Sieper J. Outcome-Parameter bei der ankylosierenden Spondylitis. Z Rheumatol 2006; 65: 131-138

6 Horn K, Gülich M, Lay W et al. Problemorientiertes Lernen (POL) in der Rehabilitation am Beispiel eines Patienten mit Spondylitis ankylosans. Rehabilitation 2007; 46: 164-174

7 Steiner WA, Ryser L, Huber E et al. Use of the ICF model as a clinical problem-solving tool in physical therapy and rehabilitation medicine. Phys Ther 2002; 82: $1098-1107$

8 Lautenschläger J, Mau W, Kohlmann T et al. Vergleichende Evaluation einer deutschen Version des Health Assessment Questionnaires (HAQ) und des Funktionsfragebogens Hannover (FFbH). Z Rheumatol 1997; 56: $144-155$

9 Streibelt M, Gerwinn H, Hansmeier T et al. SIMBO: Ein Screening-Instrument zur Feststellung des Bedarfs an Medizinisch-Beruflich Orientierten Maßnahmen in der medizinischen Rehabilitation - Analysen zur Konstruktvalidität und Prognosegüte. Rehabilitation 2007; 46: 266-275

10 Rudwaleit M, Haibel H, Baraliakos X et al. The early disease stage in axial spondylarthritis: Results from the German Spondyloarthritis Inception Cohort. Arthritis Rheum 2009; 60: 717-727

11 Huscher D, Merkesdal S, Thiele K et al. Cost of illness in rheumatoid arthritis, ankylosing spondylitis, psoriatic arthritis and systemic lupus erythematosus in Germany. Ann Rheum Dis 2006; 65: 1175- 1183

12 Mau W, Listing J, Huscher D et al. Employment across chronic inflammatory rheumatic diseases and comparison with the general population. J Rheumatol 2005; 32: $721-728$

13 van Tubergen $A$, Landewé $R$, van der Heijde $D$ et al. Combined spa-exercise therapy is effective in patients with ankylosing spondylitis: a randomized controlled trial. Arthritis Rheum 2001; 45: 430-438

14 van Tubergen A, Boonen A, Landewé $R$ et al. Cost effectiveness of combined spa-exercise therapy in ankylosing spondylitis: A randomized controlled trial. Arthritis Rheum 2002; 47: 459-467

15 Staalesen Strumse YA, Nordvåg BY, Stanghelle JK et al. Efficacy of rehabilitation for patients with ankylosing spondylitis: Comparison of a four-week rehabilitation programme in a Mediteranean and a Norwegian setting. J Rehabil Med 2011; 43: 534-542

16 Masiero S, Bonaldo L, Pigatto $M$ et al. Rehabilitation Treatment in Patients with Ankylosing Spondylitis Stabilized with Tumor Necrosis Factor Inhibitor Therapy. A Randomized Controlled Trial. J Rheumato 2011; 38: 1335-1342

17 Deutsche Rentenversicherung. Anforderungsprofil zur Durchführung der Medizinisch-beruflich orientierten Rehabilitation (MBOR) im Auftrag der Deutschen Rentenversicherung - Somatische Indikationen. 2010

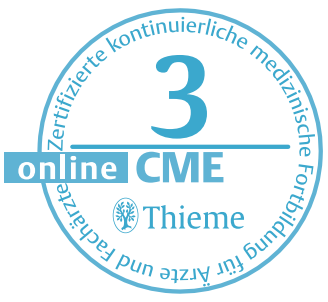

18 Barkham N, Coates LC, Keen H et al. Double-blind placebo-controlled trial of etanercept in the prevention of work disability in ankylosing spondylitis. Ann Rheum Dis 2010; 69: 1926-1928

19 Maksymowych WP, Gooch KL, Wong RL et al. Impact of Age, Sex, Physical Function, Health-related Quality of Life, and Treatment with Adalimumab on Work Status and Work Productivity of Patients with Ankylosing Spondylitis. J Rheumatology 2010; 37: 385-392

20 Braun J, van den Berg $R$, Baraliakos $X$ et al. 2010 update of the ASAS/EULAR recommendations for the management of ankylosing spondylitis. Ann Rheum Dis 2011; 70: 896-904

21 Colina M, Ciancio G, Garavini $R$ et al. Combination treatment with etanercept and an intensive spa rehabilitation program in active ankylosing spondylitis. Int J Immunopathol Pharmacol 2009; 22: 1125 1129

22 Dagfinrud H, Hagen KB, Kvien TK. Physiotherapy interventions for ankylosing spondylitis. The Cochrane Library 2009: 1-83

23 Lange U. Differenzialindikative physikalisch-medizinische Verordnung bei ankylosierender Spondylitis unter Berücksichtigung evidenzbasierter Daten. Phys Rehab Kur Med 2012; 22: 35-44

24 Mattukat K, Rennert D, Thyrolf A, Mau W. ABSCHLUSSBERICHT „Reha-Optimierung bei Patienten mit Polyarthritis oder Spondyloarthritis durch ein individuell vereinbartes Kraft, Ausdauer und Koordinationstraining (KAKo-Training)“. (22.09.2011). Im Internet: http://www.forschung-patientenorientie rung.de/files/kako-abschlussbericht.pdf, Stand: 02.03.2012

25 Bönisch A, Ehlebracht-König I. Die Veränderung des Bewegungsverhaltens nach der medizinischen Rehabilitation bei Patienten mit Spondyloarthritiden, chronischen Polyarthritiden und Fibromyalgie-Syndrom. Akt Rheumatol 2006; 31: 75-82

26 Spadaro A, de Luca T, Massimiani MP et al. Occupational therapy in ankylosing spondylitis: Short-term prospective study in patients treated with anti-TNFalpha drugs. Joint Bone Spine 2008; 75: 29-33

27 Gurcay E, Yuzer S, Eksioglu E et al. Stanger bath therapy for ankylosing spondylitis: illusion or reality? Clin Rheumatol 2008; 27: 913-917

28 Bengel J, Helmes A. Rehabilitation. In: Perrez M, Baumann U, Hrsg. Lehrbuch klinische Psychologie - Psychotherapie. 4. Aufl. Bern: Huber; 2011: 530-553

29 Barlow JH, Macey S, Struthers GR. Health Locus of Control, Self-Help and Treatment Adherence in Relation to Ankylosing Spondylitis Patients. Patient Educ Couns 1993; 20: 153-166

30 Günther V, Mur E, Traweger $C$ et al. Stress coping of patients with ankylosing spondylitis. J Psychosom Res 1994; 38: 419-427

31 Martindale J, Smith J, Sutton CJ et al. Disease and psychological status in ankylosing spondylitis. Rheumatology 2006; 45: 1288 - 1293

32 Baysal Ö, Durmuş B, Ersoy Y et al. Relationship between psychological status and disease activity and quality of life in ankylosing spondylitis. Rheumatol Int 2011; 31: 795-800

33 Faller H, Reusch A, Meng K. DGRW-Update: Patientenschulung. Rehabilitation 2011; 50: 284-291

34 Ehlebracht-König I, Bönisch A. Patientenschulung verändert Einstellungen, Funktionskapazität und sozialmedizinische Ergebnisse - Schulungsgestaltung und Evaluationsergebnisse am Beispiel der Spondylitis ankylosans. In: Petermann F, Ehlebracht-König I, Hrsg. Patientenmotivation und Compliance. Regensburg: Roderer; 2007: 151-163

35 Bönisch A, Ehlebracht-König I, Krauth C, Rieger J. Evaluation eines Schulungsprogramms für Patienten mit Spondylitis ankylosans. In: Petermann F, Hrsg. Prädiktion, Verfahrensoptimierung und Kosten in der medizinischen Rehabilitation. Regensburg: S. Roderer Verlag; 2005: 51-102

36 Krauth C, Rieger J, Bönisch A, Ehlebracht-König I. Gesundheitsökonomische Evaluation eines Patientenschulungsprogramms Spondylitis ankylosans in der stationären Rehabilitation. In: Petermann F, Hrsg. Prädiktion, Verfahrensoptimierung und Kosten in der medizinischen Rehabilitation. 2. Aufl. Regensburg: Roderer; 2005: 103-143

37 Ward MM, Kuzis S. Risk factors for work disability in patients with ankylosing spondylitis. J Rheumatol 2001; 28: 315-321

38 Bethge M. Erfolgsfaktoren medizinisch-beruflich orientierter orthopädischer Rehabilitation. Rehabilitation 2011; 50: 145-151 


\section{CME-Fragen Rehabilitation bei axialen Spondyloarthritiden}

1 Welche Merkmalkombination ist Bestandteil der heute anzuwendenden ASAS-Klassifikationskriterien der axialen Spondyloarthritiden (SpA)?

A akuter Rückenschmerz $<6$ Wochen bei Beginn $<30$ Jahre

B weibliches Geschlecht und Nachweis von CCP-Antikörpern

C Syndesmophyten der Lendenwirbelsäule und erhöhte BSG (Blutkörperchensenkungsgeschwindigkeit nach Westergren)

D chronische Rückenschmerzen $\geq 3$ Monate bei Beginn $<45$ Jahre

E ausstrahlender Kreuzschmerz bis zur Ferse bei Beginn $<40$ Jahre

2 Welches Kriterium ist in den heute anzuwendenden ASASKlassifikationskriterien der SpA neu gegenüber früheren Kriterien?

A der erhöhte Finger-Boden-Abstand

B die Sakroiliitis in der Magnetresonanztomografie

C die eingeschränkte Lendenwirbelsäulenbeweglichkeit

D die reduzierte Atembreite

E der reduzierte Hämoglobinwert

3 Welches Assessment-Instrument ist bei SpA besonders geeignet, um alltagsrelevante Aktivität als Teilaspekt der Rehabilitationsindikation entsprechend der ICF (Internationale Klassifikation der Funktionsfähigkeit, Behinderung und Gesundheit) zu erfassen?

A der Bath Ankylosing Spondylitis Metrology Index (BASMI)

B die Hospital Anxiety and Depression Scale (HADS)

C die Arbeitsunfähigkeitsdauer

D das Schober'sche Maß

E der Bath Ankylosing Spondylitis Functional Index (BASFI)

4 Welche typischen Merkmale kennzeichnen das Team für die stationäre oder ganztags ambulante Rehabilitation von Menschen mit SpA?

A die interdisziplinäre Zusammensetzung und Erfahrung mit SpA

B die Koordination durch einen Allgemeinarzt

C die Orientierung v.a. an der ICD (Internationale Klassifikation der Krankheiten)

D die Steuerung durch einen Arbeitsmediziner

E die Koordination durch einen Reha-Fachberater

5 Welche Aussage zu Interventionen in der Rehabilitation von Menschen mit SpA trifft zu?

A Die Überprüfung der medikamentösen Therapie ist von untergeordneter Bedeutung.

B Die medizinisch-berufliche Orientierung soll durch die Leistungsträger nach der Entlassung veranlasst werden.

C Krankheitsangepasste möglichst intensive Bewegungsübungen sind von zentraler Bedeutung.

D Bei Abneigungen gegen Bewegungsaktivitäten sollten ausschließlich entspannende Verfahren eingesetzt werden.

E Psychologische Interventionen sind nicht indiziert.
6 Welche Aussage zur medikamentösen Therapie der SpA in der Rehabilitation ist richtig?

A Jeder Rehabilitand mit einer SpA sollte heute mit Biologika - v. a. TNF- $\alpha$-Blocker - behandelt werden.

B Zu Beginn der Rehabilitation sollte ein Auslassversuch aller Rheumamedikamente unternommen werden.

C Die bisherigen Rheumamedikamente sollten i.d.R. durch Phytotherapeutika ersetzt werden.

D Nichtsteroidale Antirheumatika (NSAR) sind die wichtigste Substanzgruppe.

E Bei erhöhten laborchemischen Entzündungszeichen sollten primär Kortisonpräparate verabreicht werden.

7 Welche Aussage zur physikalischen Therapie der SpA in der Rehabilitation ist richtig?

A Physikalische Maßnahmen tragen zur Schmerzreduktion bei.

B Für die Durchführung von Bewegungsübungen hat die physikalische Therapie keine Bedeutung.

C Es gibt keine Hinweise auf die Wirksamkeit von Stangerbädern.

D Heiße Moorbäder sind auch bei entzündlich hochaktiver SpA unverzichtbar.

E Der Stellenwert der Elektrotherapie ist in den letzten 10 Jahren gestiegen.

8 Welche Aussage zur Patientenschulung von Menschen mit entzündlicher Wirbelsäulenerkrankung in der Rehabilitation ist richtig?

A Es gibt kein spezifisches Schulungsprogramm für Patienten mit entzündlicher Wirbelsäulenerkrankung.

B Standardisierte Schulungen für Patienten mit entzündlicher Wirbelsäulenerkrankung sollten von einer Berufsgruppe durchgeführt werden.

C Nur kognitive Elemente sollten Gegenstand der Patientenschulung von Menschen mit entzündlicher Wirbelsäulenerkrankung sein.

D Ein standardisiertes Schulungsprogramm verbessert die Selbstwirksamkeit und Handlungskompetenz.

E Emotionale Aspekte sollten in Patientenschulung von Menschen mit entzündlicher Wirbelsäulenerkrankung nicht thematisiert werden.

9 Welche Aussage zur Planung nach der Entlassung von Menschen mit SpA aus der Rehabilitationseinrichtung trifft zu?

A Weiterführende Maßnahmen oder Empfehlungen sind in der Regel nicht erforderlich.

B In der Klinik können Nachsorgeleistungen wie Funktionstraining oder Rehabilitationssport verordnet werden.

C Leistungen zur Teilhabe am Arbeitsleben sind nicht von Bedeutung.

D Stufenweise Wiedereingliederung am Arbeitsplatz ist bei arbeitsfähig entlassenen Rehabilitanden einzuleiten.

E Eine Information der weiterbetreuenden Ärzte zur Einleitung einer Biologika-Therapie ist nicht zielführend. 
10

A

vom Arzt festzulegen.

B Allgemein formulierte Ziele sind für die Gestaltung des Rehabilitationsprozesses am besten geeignet.

C Individuelle Verhaltensänderungen gehören nicht zu den Rehabilitationszielen.

D Vordergründiges Ziel ist die Erholung durch die Kombination aus Bädern und langen therapiefreien Intervallen.

E Die Heranführung und Bindung der Betroffenen an regelmäßige körperliche Aktivität gehört zu den wichtigsten Zielen der Rehabilitation. 